Journal of Integrative Neuroscience, Vol. 11, No. 1 (2012) 17-32

(c) Imperial College Press

DOI: $10.1142 / \mathrm{S} 0219635212500021$

\title{
Serial pattern learning during skilled walking
}

\author{
Douglas G. Wallace and Shawn S. Winter \\ Psychology Department \\ Northern Illinois University \\ Dekalb, Illinois USA 60115 \\ Gerlinde A. Metz \\ Canadian Centre for Behavioural Neuroscience \\ University of Lethbridge \\ Lethbridge, Alberta Canada T1K 3M4
}

[Received 5 January 2011; Accepted 9 September 2011]

\begin{abstract}
Rats possess a rich repertoire of sequentially organized, natural behaviors. It is possible that these natural behaviors may reflect implicit learning or relatively fixed movement patterns. The present study was conducted to determine whether factors known to influence implicit learning produce similar effects on the acquisition of skilled walking. Three groups of rats were trained to cross a horizontal ladder with rungs spaced according to three different levels of complexity. All training and testing were performed under dark conditions to assess the influence of non-visual modalities on skilled walking. Although all groups' performance improved throughout training, pattern complexity influenced the rate of improvement. In addition, performance during a probe session provided further evidence that each group encoded the rung spacing pattern experienced during training to create an internal representation. These observations demonstrate that the engram established during repetitive training represents either the temporal or spatial characteristics of rung spacing. These findings indicate that implicit learning contributes to the acquisition of natural sequential behaviors. Furthermore, serial pattern learning of rung spacing provides a novel task to determine sensory and motor contributions to the consolidation of skilled movement.
\end{abstract}

Keywords: Rattus norvegicus; motor; sequential; rule; implicit; associative processes.

\section{Introduction}

Animal behavior is sequentially organized. Animals encode frequently experienced sequences of stimuli or responses. This sequential learning is an example of implicit learning that has traditionally been investigated using operant procedures. Studies have demonstrated a number of factors that influence this type of implicit learning. For example, rats learn to vary running speed depending on the amount of food that is anticipated at the end of a runway. Provided that changes in reward magnitude across trials are monotonic (i.e., decreasing), rats' anticipatory learning is facilitated; however, non-monotonic (i.e., both decreasing and increasing) changes in reward magnitude across trials are associated with attenuated anticipatory learning 
(Hulse \& Dorsky, 1977). Similar effects of pattern complexity have been observed in rats learning to respond to a circular array of eight levers (Fountain \& Rowan, 1995). Although debate continues over the nature of the engram mediating sequential learning in these tasks, associative mechanisms appear sufficient to account for many aspects of the behavior (Capaldi \& Molina, 1979; Wallace et al., 2008; Wallace \& Fountain, 2002). Recent progress has been made in understanding the neural basis of these forms of implicit learning and their internal representation (Hübener \& Bonhoeffer, 2010).

Rats engage in a variety of natural behaviors that also appear to be sequentially organized. For example, rats are able to manipulate small objects or to navigate across difficult territory using sequences of discrete limb and digit movements (Whishaw, 2005; Metz \& Whishaw, 2002). Relatively more is known about the contribution of specific neural structures to the organization of these natural sequential behaviors. Studies have demonstrated that both unilateral motor cortex (Whishaw, 2000) and nigrostriatal bundle lesions (Metz et al., 2001) disrupt skilled reaching performance. These disruptions in performance associated with motor cortex lesions have been attributed to a loss of the representation for the sequence of movements, or the motor engram (Whishaw, 2000). Once damaged, the motor engram comprising the original movement sequence remains, at least in part, permanently disrupted (Whishaw, 2000; Metz \& Whishaw, 2002). Thus, it has been suggested that improvements in performance subsequent to motor cortex lesions are mediated by the emergence of a new sequence of movements (Whishaw, 2000; Metz et al., 2005; Gharbawie \& Whishaw, 2006), which is also reflected in the rearrangement of cortical motor maps (Monfils et al., 2005). New movement sequences primarily involve the use of compensatory strategies based on proximal limb movement and body posture (Whishaw et al., 1991; Whishaw, 2000; Metz et al., 2005). Relatively less is know about the nature of the engram mediating these natural sequential behaviors. It is not clear which proportion of these natural behaviors reflect implicit learning or relatively fixed movement patterns (Metz \& Whishaw, 2002).

The goal of the present study was to investigate whether factors known to influence implicit learning in traditional operant tasks produce similar effects on the acquisition of a natural sequential behavior. Previous studies have demonstrated a considerable capacity for rats to modify their gait patterns to move across difficult terrains (Metz \& Whishaw, 2002; Bolton et al., 2006). The current study examined the capacity for rats to acquire skilled walking under conditions of varied complexity. Terrain complexity was varied by manipulating the spacing between rungs on the horizontal ladder apparatus. Three groups of rats experienced different patterns of rung spacing that varied in complexity. Subsequent to training, all groups were exposed to a novel sequence of rung spacing that varied in similarity to the sequence experienced during training. Observing that performance improves during training, that complexity of rung spacing influences the rate of improvement, and that modification of rung spacing disrupts performance would provide evidence 
that animals were encoding the sequential characteristics of the rung spacing. In addition, this study examined whether systematic variation of sequential characteristics differentially influenced acquisition of skilled walking by forelimbs and hind limbs. The results of this study will determine the extent that a natural sequential behavior, skilled walking, is mediated by implicit learning.

\section{Materials and methods}

\subsection{Subjects}

Subjects were 12 naïve female Long Evans rats. Animals were raised and housed at the University of Lethbridge vivarium in wire mesh cages in groups of two. The light cycle was 12:12 h with light starting at 8:00 AM, and the room temperature was maintained at $22^{\circ} \mathrm{C}$. Subsequent to daily testing, rats were fed rat chow to maintain them at $85 \%$ of their ad lib weight.

\subsection{Apparatus}

The rung walking apparatus consisted of two clear Plexiglas walls $(100 \mathrm{~cm} \times 20 \mathrm{~cm})$ held together by 100 equally spaced metal rungs (3-mm diameter) inserted through the bottom of each wall (see Fig. 1). The apparatus spanned two platforms such that the bottom was elevated $30 \mathrm{~cm}$ above the ground. The apparatus was located in a large room that could be made completely dark. The experimenter used an infrared spotter to monitor the rat's behavior during testing. A camera equipped to record under dark conditions (sensitive to infrared wavelengths) was positioned perpendicular to the longer side of the apparatus. Alley width was adjusted to the size of the animal so that it was about $1 \mathrm{~cm}$ wider than an animal, thereby preventing the animal from turning around.

The spacing between rungs could be increased by removal of adjacent rungs. Removal of one, two, or three rungs resulted in 2-cm, 3-cm, or 4-cm gaps, respectively. Serial patterns were produced by systematically varying the spacing between rungs across the entire apparatus. Four different patterns were used in the current study. First, the standard pattern reflected the removal of every other rung. This sequence of rung spacing reflects the repeated application of an identity rule.

Standard: 11111111111111111111111111111111111111111 11111111

Second, the two-level pattern involved a nesting of two rules. The lower level rule involves increasing the number of rungs removed. The higher level rule involves resetting to one rung spacing after two applications of the lower level rule.

Two-level: 123123123123123123123123123123123

Third, one of the three-level patterns (used during training only) started the same as the two-level pattern; however, approximately halfway through the sequence, a third level rule (underlined items) was applied that mirrored the pattern of rung spacing previously encountered. 


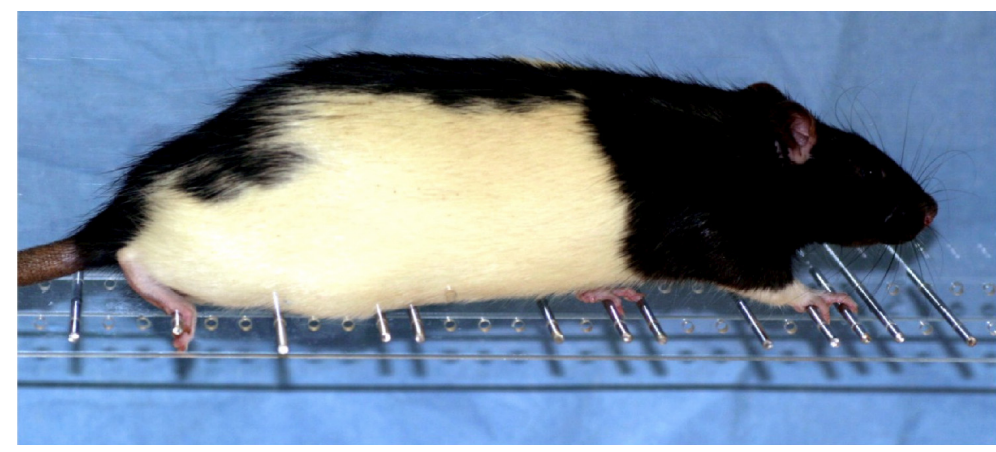

(a)

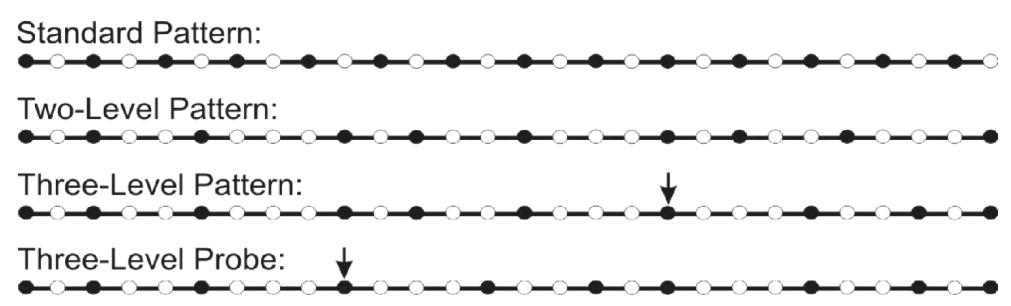

(b)

Fig. 1. Photograph of a rat's forelimb and hind limb place while crossing the horizontal ladder apparatus. Rung spacing sequences were created by varying the number rungs removed: Standard (111111), Two-level (123123), and Three-level (123321). The probe sequence was similar to the three-level pattern experienced during training except the third level rule transition occurred earlier in the sequence.

Three-level: $123123123123123123 \underline{321321321321321}$

Finally, a different three-level pattern was reserved for the probe session. This pattern was the same as the three-level pattern previously described except the third level rule transition (underlined items) occurred earlier in the sequence.

Probe: $123123123 \underline{321321321321321321321321}$

\subsection{Procedure}

All behavioral procedures were conducted under complete dark conditions. Rats were individually placed at one end of the apparatus and were allowed to traverse the rungs to obtain three 45-mg banana food pellets (Bio-Serve Inc., Frenchtown, NJ) at the far end of the apparatus. All rats crossed the apparatus in the same direction. Upon consumption of the food pellets, the rat was placed back at the starting location. This continued until the rat completed three trials. Behavior testing involved three phases: pre-training, training, and probe.

During the pre-training phase, all rats were exposed to the standard pattern for five days. This habituated the rats to the apparatus and associated crossing the rungs with obtaining food pellets.

Rats were divided into three groups based on the rung sequence experienced throughout the 14 days of training. Group $111111(n=4)$ was trained with the 
standard pattern. Group $123123(n=4)$ was trained with the two-level pattern. Group $123321(n=4)$ was trained with the three-level pattern.

On day 20, all rats were exposed to the three-level probe pattern. For the standard pattern group (i.e., 111111), rats experienced two additional levels of rule organization during this probe trial, whereas the two-level group (i.e., 123123) experienced only one additional level of rule organization during the probe. For group 123321, the probe only modified the location of the third-level rule transition.

\subsection{Analysis of skilled walking}

Several characteristics of behavior were used to assess performance during each phase of testing. First, the number of errors observed across the three trials was calculated for each day. Errors were defined according to the rating scale developed by (Metz \& Whishaw, 2002). In the current study, slight slips, deep slips, or total misses of fore- and hind limbs were counted as errors. Group differences were evaluated with ANOVAs conducted on total errors per day.

Another characteristic of behavior used to quantify performance was the latency to transverse the apparatus. A trial was initiated when both hind limbs entered the alley and concluded when both hind limbs exited the alley. Group differences were evaluated with ANOVAs conducted on average daily latency.

Finally, the number of errors associated with forelimb (FLE) and hind limb (HLE) placement was recorded for each trial. Group differences in daily percent of forelimb errors [(FLE/FLE+HLE)*100] were evaluated using ANOVAs.

\section{Results}

\subsection{Pre-training}

Total number of errors decreased during pre-training with the standard pattern (see Fig. 2(a)). The ANOVA conducted on daily errors revealed a significant main effect of days $\left[\mathrm{F}(4,44)=6.863, p<0.001, \eta_{p}^{2}=0.384\right]$. A significant linear trend $[\mathrm{F}$ $\left.(1,11)=7.626, p=0.019, \eta_{p}^{2}=0.409\right]$ in daily errors was observed across the five days of pre-training.

Daily latencies became shorter during pre-training with the standard pattern (see Fig. 2(b)). The ANOVA conducted on average daily latency revealed a significant effect of day $\left[\mathrm{F}(4,44)=6.166, p<0.001, \eta_{p}^{2}=0.359\right]$. A significant linear trend $\left[\mathrm{F}(1,11)=25.532, p<0.001, \eta_{p}^{2}=0.699\right]$ in daily latencies was observed across the five days of pre-training.

Daily errors were evenly distributed across forelimbs and hind limbs (see Fig. 2(c)). This distribution did not change across days. The ANOVA conducted on daily percent forelimb errors failed to reveal a significant effect of days $[\mathrm{F}(4,44)=1.594$, $\left.p=0.193, \eta_{p}^{2}=0.127\right]$. The average percent of forelimb errors across all days of pretraining was 52.048 . 


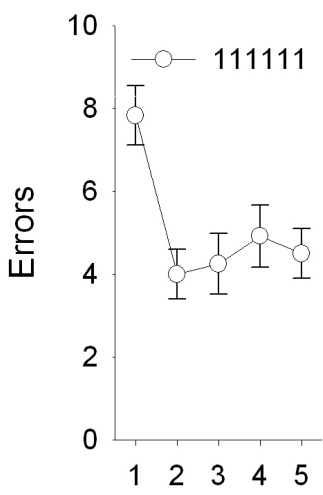

(a)

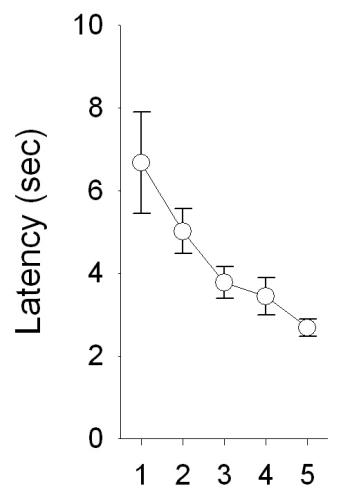

(b)

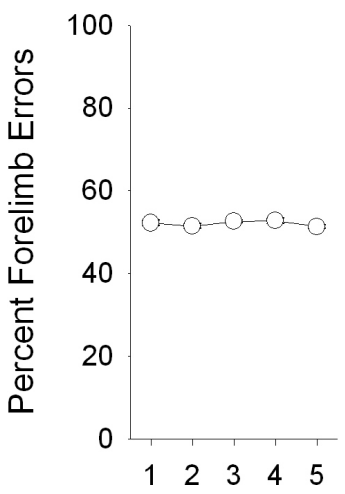

(c)

Fig. 2. All rats' (a) average total number of errors, (b) latency to cross the apparatus, and (c) percent forelimb errors, are plotted for the five sessions of pre-training. Note: during pre-training all rats were exposed to the Standard sequence of rung spacing.

\subsection{Training}

During training, each group experienced a different rung spacing sequence. Each group's number of errors gradually decreased across training days (see Fig. 3(a)). The ANOVA conducted on daily errors revealed significant main effects of group $\left[\mathrm{F}(2,9)=4.392, p=0.047, \eta_{p}^{2}=0.494\right]$ and day $[\mathrm{F}(13,117)=2.714, p=0.002$, $\left.\eta_{p}^{2}=0.232\right]$; however, the Group by Day $\left[\mathrm{F}(26,117)=0.724, p=0.828, \eta_{p}^{2}=0.139\right]$ interaction was not significant. Post-hoc analyses revealed that only the group trained with the three-level pattern made significantly more errors than the group trained with the two-level pattern (LSD, $p<0.05$ ). A significant linear trend $\left[\mathrm{F}(1,9)=17.926, p=0.002, \eta_{p}^{2}=0.666\right]$ in daily errors was observed across days.

Each group's daily latencies were observed to decrease throughout the training phase (see Fig. 3(b)). The ANOVA conducted on average daily latency revealed significant main effects of group $\left[\mathrm{F}(2,9)=4.731, p=0.039, \eta_{p}^{2}=0.513\right]$ and

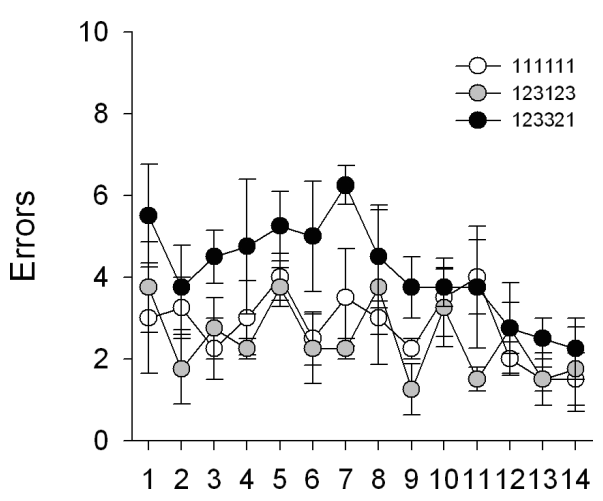

(a)

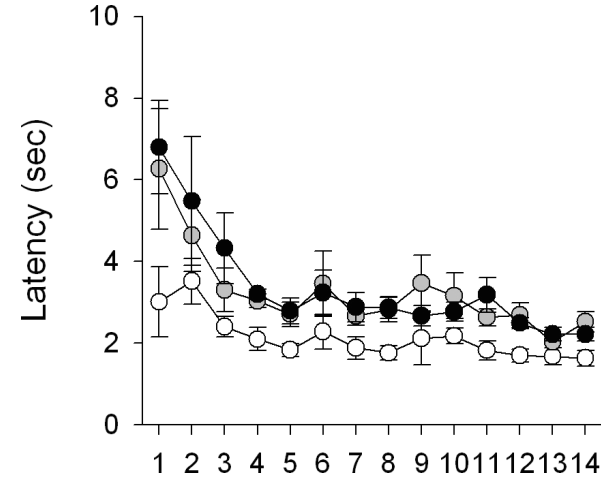

(b)

Fig. 3. Each group's (a) average total number of errors, (b) latency to cross the apparatus, and (c) percent forelimb errors, are plotted for the 14 days of training. 


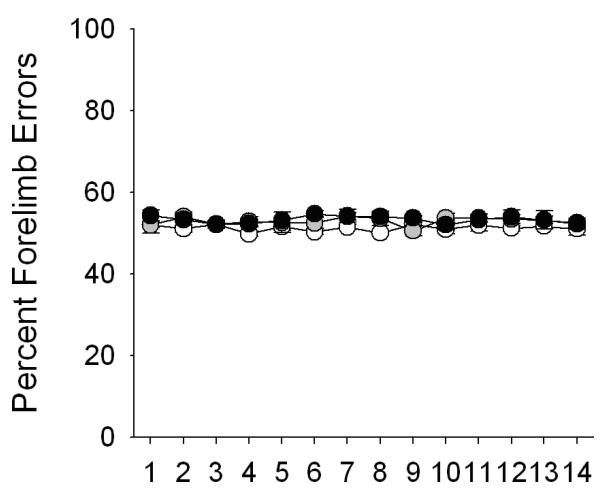

(c)

Fig. 3. (Continued)

days $\left[\mathrm{F}(13,117)=11.895, p<0.001, \eta_{p}^{2}=0.569\right]$; however, the Group by Days $\left[\mathrm{F}(26,117)=1.067, p=0.391, \eta_{p}^{2}=0.192\right]$ interaction was not significant. Post-hoc analyses revealed that the group trained with the standard pattern took significantly shorter times to traverse the apparatus than the other two groups (LSD, $p<0.05$ ). A significant linear trend $\left[\mathrm{F}(1,9)=35.838, p<0.001, \eta_{p}^{2}=0.799\right]$ in daily latencies was observed across days.

Throughout training, each group's daily errors were evenly distributed across the forelimbs and hind limbs (see Fig. 3(c)). The ANOVA conducted on daily percent forelimb errors failed to reveal a significant effect of group $[\mathrm{F}(2,9)=3.031$, $\left.p=0.099, \eta_{p}^{2}=0.402\right]$, days $\left[\mathrm{F}(13,117)=0.524, p=0.906, \eta_{p}^{2}=0.055\right]$, and Group by Days $\left[\mathrm{F}(26,117)=0.809, p=0.792, \eta_{p}^{2}=0.152\right]$ interaction. The overall average percent of forelimb errors across all days was 52.491 .

\subsection{Day 14 vs. probe}

By day 14 of training, all rats were at asymptotic levels of performance. Significant increases in errors were associated with transferring each group to the three-level probe pattern (see Fig. 4(a)). The ANOVA conducted on daily errors revealed a significant main effect of day $\left[\mathrm{F}(1,9)=33.379, p<0.001, \eta_{p}^{2}=0.788\right]$; however, the main effect of group $\left[\mathrm{F}(2,9)=0.626, p=0.556, \eta_{p}^{2}=0.122\right]$ and the Group by Day $\left[\mathrm{F}(2,9)=1.155, p=0.358, \eta_{p}^{2}=0.358\right]$ interaction were not found to be significant.

Latency to cross the apparatus only increased during the probe for the group trained on the standard pattern (see Fig. 4(b)). The ANOVA conducted on average daily latencies revealed a significant main effect of day $[\mathrm{F}(1,9)=9.775$, $\left.p=0.012, \eta_{p}^{2}=0.521\right]$ and Group by Day $\left[\mathrm{F}(2,9)=8.249, p=0.009, \eta_{p}^{2}=0.647\right]$ interaction; however, the main effect of group $[\mathrm{F}(2,9)=1.761, \quad p=0.226$, $\left.\eta_{p}^{2}=0.281\right]$ was not found to be significant. Post-hoc analyses revealed that although latencies did not differ among groups on the last day of training, the 


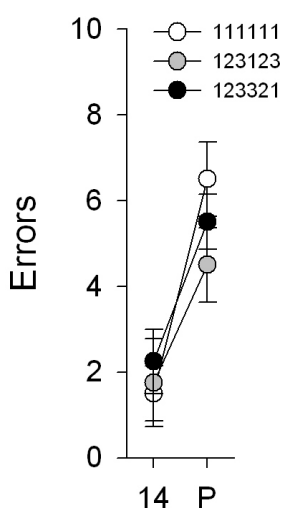

(a)

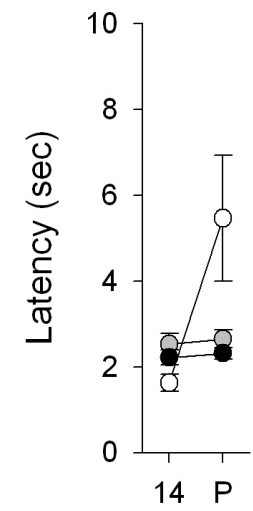

(b)

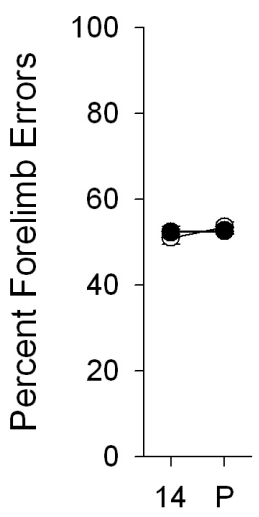

(c)

Fig. 4. Each group's (a) average total number of errors, (b) latency to cross the apparatus, and (c) percent forelimb errors, are plotted for the last day of training and the probe session.

group trained with the standard sequence had significantly slower latencies relative to the other groups on the probe day (LSD, $p<0.05$ ).

All groups' daily errors were evenly distributed across forelimbs and hind limbs during the last day of training and the probe session (see Fig. 4(c)). The ANOVA conducted on daily percent forelimb errors failed to reveal a significant main effect of group $\left[\mathrm{F}(2,9)=0.026, p=0.974, \eta_{p}^{2}=0.006\right]$, days $[\mathrm{F}(1,9)=1.948$, $\left.p=0.196, \eta_{p}^{2}=0.178\right]$, and Group by Days $\left[\mathrm{F}(2,9)=1.016, p=0.400, \eta_{p}^{2}=0.184\right]$ interaction. The overall average percent of forelimb errors across both days was 52.450 .

\section{Discussion}

The current study investigated the effects of rung spacing organization on acquisition of skilled walking. Several aspects of behavior were consistent with rats encoding the sequence of rung spacing to guide performance. First, both error rates and latencies were found to decrease throughout training. Although consistent with rats learning the sequential organization of rung spacing, this may only reflect rats encoding non-sequential aspects of the task (e.g., motivational demands). Second, group differences in performance were observed during training. Considering that only pattern complexity varied among groups, differences in performance were not dependent on non-sequential aspects of the task. Finally, an increase in errors was observed during the probe session in which all rats were exposed to a new rung spacing sequence. These results are consistent with a conflict between the motor skills acquired during training and the new rung spacing sequence experienced during the probe. These observations demonstrate that rats encode the sequential characteristics of rung spacing to guide skilled walking on the horizontal ladder apparatus. 


\subsection{Nature of the engram}

Debate has focused on the nature of the engram mediating sequential behavior. Two theories have been advanced to explain how animals organize their behavior through time. One view contends that animals encode the symbolic relationships or rules that describe a set of stimuli or responses (Hulse \& Dorsky, 1977). For example, rats learned to modify their runway latencies based on the anticipated magnitude of reward. The rate of learning was shown to depend on the number of rules required to relate items of the set (Hulse \& Dorsky, 1977). Reward magnitude sequences that can be described with one rule (i.e., monotonic: 14-7-3-1-0) are learned more quickly relative to sequences that require multiple rules (i.e., non-monotonic: 14-1-3-7-0). In addition, temporal presentation of sequential items or phrasing that is consistent with the rule structure facilitates sequential learning, whereas phrasing that is inconsistent with the rule structure impairs sequential learning (Fountain et al., 1984). Although these observations are consistent with rats encoding the rule structure of a set of stimuli to guide performance, debate has focused on whether simpler processes may be sufficient to explain these characteristics of sequential behavior.

The other predominant theory posits that rats encode the associations between recently experienced reward magnitudes (Capaldi \& Molina, 1979). Under this view, the rate of sequential learning depends on stimulus discriminability. Highly discriminable, non-monotonic sequences (i.e., 1-29-0) of reward magnitude have been shown to be learned faster relative to less discriminable, monotonic sequences (i.e., 20-10-0) of reward magnitude. Furthermore, phrasing manipulations have been shown to influence item discriminability (Capaldi et al., 1984). Phrasing cues act to overshadow associations between items that are of low discriminability. The associative view has subsequently been shown to accurately predict performance in food reward magnitude studies originally motivated from either view (Wallace \& Fountain, 2002).

The debate over the nature of the engram mediating sequential behavior has continued in studies examining long sequences of operant responses (Fountain \& Rowan, 1995). Using an array of eight levers (each located on a wall of an octagonal chamber) and electrical brain stimulation to maintain behavior, rats can be trained to produce sequences of 24,30 , or 36 responses. Sequence learning was shown to depend on the rule organization associated with the sequence of responses. Elements of the sequence that violated rule organization were difficult to learn and attenuated learning of the entire sequence of responses relative to a hierarchically organized sequence of the same length. In general, increasing the number of rules required to describe the sequence or pattern complexity attenuated serial pattern learning. In contrast, studies examining the effects of sequence phrasing have supported a role for phrasing cues in acting as discriminative stimuli that overshadow disruptive associations (Stempowski et al., 1999; Fountain et al., 2000; Wallace et al., 2008). Although not parsimonious, it is possible that rats may use a combination of rule and associative mechanisms to guide sequential behavior. 
Several aspects of performance during the probe session in the current study suggest that the motor engram mediating skilled walking may represent both rule and associative characteristics of the rung spacing sequence. The rung spacing encountered during the probe sequence varied in similarity to the sequences each group experienced during training. The three-level group's training sequence of rung spacing was the most similar to the probe sequence; whereas, the two-level and standard groups' training sequences were progressively more different from the probe sequence. Only the standard group exhibited a significant increase in latency to cross the apparatus during the probe session. It is possible that the two-level and three-level groups abstracted some aspect of rung organization (i.e., first and second level rules) associated with training and were able to use the engram to guide performance during the probe session. Although latency to cross the apparatus did not differ between the two-level and three-level groups, this may have reflected that both groups' performance was at asymptote or showed a floor effect.

Observing that errors increased during the probe session was consistent with a conflict between the engram acquired during training and the rung spacing sequence encountered during the probe session. The absence of group differences during the probe session provides indirect evidence that the engram may also represent associative characteristics of the sequence. Specifically, rats exposed to the threelevel pattern of rung spacing during training did not have fewer errors relative to the other groups. This was surprising, considering that this was the only group that had previous experience with a three-level pattern of rung spacing. One possible explanation is that spatial or temporal cues had become associated with the thirdlevel rule transition. Changing the location of the third level rule transition modified these cues; therefore, rats could not effectively use the engram acquired during training. Although the data of the current study demonstrate that rats encode the rung spacing sequence, further work is required to determine the nature of the engram that mediates skilled walking.

\subsection{Neural basis of the engram}

Taken together, the present findings indicate that the serial pattern learning of rung spacing provides a novel, sensitive task to determine sensory and motor contributions to skilled movement. It has been proposed that structures involved in implicit learning of motor sequences, such as the cerebellum, might operate by learning to prepare responses to predictable sensory events (Nixon \& Passingham, 2001). The repetitive patterns of rung arrangement might facilitate the acquisition and generalization of one pattern to another when animals learn to predict the spatial location of consecutive rungs.

Several lines of evidence have suggested an involvement of sensorimotor cortex in skilled movement. First, motor cortex lesions have been shown to impair both skilled reaching (Whishaw, 2000) and skilled walking (Metz \& Whishaw, 2002). 
Although performance has been shown to improve with post-surgery training, the sequence of movements reflects a concatenation of compensatory movements rather than restoration of the original skilled movement (Whishaw, 2000; Metz et al., 2005; Alaverdashvili \& Whishaw, 2010). Second, skilled reaching training can change the motor map of forelimb region representation of the intact motor cortex (Kleim et al., 1998). This reorganization appears to depend on the acquisition of sequenced skilled movement. Neither motivational aspects of reaching (Kleim et al., 2004) nor strength training (Remple et al., 2001; Kleim et al., 2002) have been demonstrated to significantly reorganize the motor cortex map. Manipulation of the sequence of rung spacing may provide a novel technique to further investigate the role of the motor cortex in acquisition of skilled movement.

Voluntary control of skilled walking requires the integrity of subcortical structures as well as the motor cortex. The basal ganglia are essential for skilled movement and posture. Loss of striatal dopamine in rats results in reduced accuracy of skilled limb movement in reaching (Whishaw et al., 1986; Metz et al., 2001) and walking tasks (Metz \& Whishaw, 2002; Whishaw et al., 2003; Klein et al., 2009). Furthermore, the basal ganglia have been suggested to be critical in selecting motor programs (Grillner et al., 2005) and in modifying motor patterns by implicit learning of sequences (Smith \& McDowall, 2006) and motor skill (Laforce \& Doyon, 2002). In addition, it has been suggested that motor sequence learning requires a tight coupling between dopamine input to caudate nucleus and thalamo-cortical activity (Carbon et al., 2004). There also have been a number of studies comparing the influence of basal ganglia and cerebellar lesions in sequence learning tasks in patients. Most of the observations suggest a role for the cerebellum in forming sequential associations (Shin \& Ivry, 2003) mainly by implicit motor learning (Matsumura et al., 2004). In rats, the cerebellum has been implicated in learning postural tasks (Ioffe et al., 2007) and in fine-tuning of limb coordination (Molinari \& Petrosini, 1993). It is likely that the acquisition of a rung arrangement in the present study involved the co-operation of motor cortex, basal ganglia and cerebellum, which then communicate with the spinal cord.

It is possible that the spinal cord itself also contributes to the acquisition of motor patterns. Spinal plasticity has been demonstrated in conditioned reactions (Wolpaw, 1997). It can be speculated that complex gait patterns, such as those required for skilled walking, largely rely on higher order structures to place fore- and hind limbs correctly (Bolton et al., 2006). Voluntary adjustments of gait patterns are mainly mediated by the corticospinal and rubrospinal tracts (Metz \& Whishaw, 2002; Webb \& Muir, 2003). These descending pathways relay the input from motor cortex and midbrain, respectively, which might respond to the acquisition of a serial rung arrangement by rapid plasticity that in turn might gradually induce plastic changes within the spinal cord, as well (Wolpaw \& Chen, 2006). These spinal as well as supraspinal structures integrate sensory information to locate individual rungs and perform accurate limb placements. 


\subsection{The role of hapsis in skilled walking}

The current findings indicate that correct limb placement requires a variety of sensory modalities. The study was conducted under completely dark conditions, thereby eliminating the use of visual cues for limb placement. The rats were able to perform skilled limb movements in the absence of visual input to accurately place fore- and hind limbs on rungs. These findings are in line with observations showing that rats are able to maintain success of skilled reaching in the dark (Whishaw \& Tomie, 1989). While it was found that olfactory cues are necessary for locating food pellets and direct skilled reaching movements (Whishaw \& Tomie, 1989), it can be speculated that proprioceptive, haptic and vibrissa tactile sensation are more critical to locate rungs when walking. Under dark conditions, rats heavily rely on the function of sinus hairs for orienting in space and locating remote rungs. In particular, the long vibrissae located on the rat's upper lip sense small deflections to locate obstacles. These highly sensitive hairs become particularly important when palpating the surface area and locating rung positions during skilled walking. Furthermore, rats have extended tactile hairs in all regions of their skin, including the caudal aspect of the thoracic limb, proximal to the carpal joint. These hairs might be most important for fine adjustments of paw position on a rung. The importance of tactile body hairs aside from vibrissae is supported by the observation that rats with trimmed vibrissae still perform well in a skilled walking task such as crossing a horizontal rope (Prchal et al., 2004). Positioning on small objects such as a narrow beam or rope might require feedback from tactile hairs of the ventral coat, which might also contribute when adjusting body and hind limb position on the ladder rung task. The involvement of hapsis in skilled walking strengthens the notion of control of skilled walking by cortical structures.

\subsection{Sexually dimorphic movement patterns}

Movement organization has been shown to vary between male and female rats (Field \& Pellis, 2008). For example, if a rat is approached during the consumption of a food item, then the rat will engage in a lateral movement or dodge that protects the food item from being stolen (Whishaw \& Tomie, 1988). Movements associated with a dodge have been shown to differ between the sexes (Field et al., 1996) and these sexually dimorphic movement characteristics depend on gonadal hormones (Field et al., 1997, 2004). Considering the aforementioned sex differences are observed during spontaneously occurring behavior, one may expect sexually dimorphic performance in skilled walking. Previous work has shown a trend, although not significant, for male rats to exhibit more errors while crossing bars with random spacing (Jadavji \& Metz, 2008). The current study examined the effects of varying bar spacing complexity on skilled walking in female rats. Further work is needed to examine whether these results translate to male rats. The skilled walking paradigm provides a rich behavioral tool to evaluate whether sex differences are also observed with learning sequential behavior. 


\section{Conclusion}

The current study examined the effect of rung spacing organization on the acquisition of skilled walking. First, both errors and latencies decreased throughout training. Second, complexity of the rung spacing sequences influenced the rate at which performance improved. Finally, transferring rats to a novel rung spacing sequence disrupted performance. These results demonstrate that factors known to influence implicit learning similarly affect skilled walking. The present findings also provide a foundation for future studies investigating the neural basis of the engram mediating skilled movement.

\section{Acknowledgments}

We would like to thank Patricia S. Wallace and Ian Q. Whishaw for comments on previous drafts of the manuscript. We would also like to thank Felicia Drever for assistance in data analysis. This research has been supported by the Natural Sciences and Engineering Research Council of Canada (GM) and by a scholarship from the Alberta Heritage Foundation for Medical Research (GM).

\section{REFERENCES}

Adkins, D.L., Boychuk, J., Remple, M.S. \& Kleim, J.A. (2006) Motor training induces experience-specific patterns of plasticity across motor cortex and spinal cord. J. Appl. Physiol., 101(6), 1776-1782.

Alaverdashvili, M. \& Whishaw, I.Q. (2010) Compensation aids skilled reaching in aging and in recovery from forelimb motor cortex stroke in the rat. Neuroscience, 167(1), 21-30.

Bolton, D.A., Tse, A.D., Ballermann, M., Misiaszek, J.E. \& Fouad, K. (2006) Task specific adaptations in rat locomotion: Runway versus horizontal ladder. Behav. Brain. Res., 168 (2), 272-279.

Capaldi, E.J. \& Molina, P. (1979) Element discriminability as a determinant of serialpattern learning. Anim. Learn. Behav., 7, 318-322.

Capaldi, E.J., Verry, D.R., Nawrocki, T.M. \& Miller, D.J. (1984) Serial learning, interitem association, phrasing cues, interference, overshadowing, chunking, memory, and extinction. Anim. Learn. Beh., 12, 7-20.

Carbon, M., Ma, Y., Barnes, A., Dhawan, V., Chaly, T., Ghilardi, M.F. \& Eidelberg, D. (2004) Caudate nucleus: Influence of dopaminergic input on sequence learning and brain activation in Parkinsonism. Neuroimage, 21(4), 1497-1507.

Chen, X.Y., Chen, Y., Chen, L., Tennissen, A.M. \& Wolpaw, J.R. (2006) Corticospinal tract transection permanently abolishes H-reflex down-conditioning in rats. J. Neurotrauma, 23(11), 1705-1712.

Field, E.F. \& Pellis, S.M. (2008) The brain as the engine of sex differences in the organization of movement in rats. Arch. Sex. Behav., 37(1), 30-42.

Field, E.F., Whishaw, I.Q., Forgie, M.L. \& Pellis, S.M. (2004) Neonatal and pubertal, but not adult, ovarian steroids are necessary for the development of female-typical patterns of dodging to protect a food item. Behav. Neurosci., 118(6), 1293-1304. 
Field, E.F., Whishaw, I.Q. \& Pellis, S.M. (1996) A kinematic analysis of evasive dodging movements used during food protection in the rat (Rattusnorvegicus): Evidence for sex differences in movement. J. Comp. Psychol., 110(3), 298-306.

Field, E.F., Whishaw, I.Q. \& Pellis, S.M. (1997) A kinematic analysis of sex-typical movement patterns used during evasive dodging to protect a food item: The role of testicular hormones. Behav. Neurosci., 111(4), 808-815.

Fountain, S.B., Benson, A.M. \& Wallace, D.G. (2000) Number, but not rhythmicity, of temporal cues determines phrasing effects in rat serial-pattern learning. Learn. Motiv., 31, 301-322.

Fountain, S.B., Henne, D.R. \& Hulse, S.H. (1984) Phrasing cues and hierarchical organization in serial pattern learning by rats. J. Exp. Psych: Anim. Behav. Process., 10, 30-45.

Fountain, S.B. \& Rowan, J.D. (1995) Coding of hierarchical versus linear pattern structure in rats and humans. J. Exp. Psychol: Anim. Behav. Process., 21(3), 187-202.

Gharbawie, O.A. \& Whishaw, I.Q. (2006) Parallel stages of learning and recovery of skilled reaching after motor cortex stroke: "Oppositions" organize normal and compensatory movements. Behav. Brain. Res., 175(2), 249-262.

Grillner, S., Hellgren, J., Menard, A., Saitoh, K. \& Wikstrom, M.A. (2005) Mechanisms for selection of basic motor programs - roles for the striatum and pallidum. Trends Neurosci., 28(7), 364-370.

Hübener, M. \& Bonhoeffer, T. (2010) Searching for engrams. Neuron, 67, 363-371.

Hulse, S.H. \& Dorsky, N.P. (1977) Structural complexity as a determinant of serial pattern learning. Learn. Mot., 8, 488-506.

Ioffe, M.E., Chernikova, L.A. \& Ustinova, K.I. (2007) Role of cerebellum in learning postural tasks. Cerebellum, 6(1), 87-94.

Jadavji, N.M. \& Metz, G.A. (2008) Sex differences in skilled movement in response to restraint stress and recovery from stress. Behav. Brain. Res., 195(2), 251-259.

Kleim, J.A., Barbay, S. \& Nudo, R.J. (1998) Functional reorganization of the rat motor cortex following motor skill learning. J. Neurophysiol., 8, 3321-3325.

Kleim, J.A., Cooper, N.R. \& VandenBerg, P.M. (2002) Exercise induces angiogenesis but does not alter movement representations within rat motor cortex. Brain. Res., 934(1), $1-6$.

Kleim, J.A., Hogg, T.M., VandenBerg, P.M., Cooper, N.R., Bruneau, R. \& Remple, M. (2004) Cortical synaptogenesis and motor map reorganization occur during late, but not early, phase of motor skill learning. J. Neurosci., 24(3), 628-633.

Kleim, J.A., Vij, K., Ballard, D.H. \& Greenough, W.T. (1997) Learning-dependent synaptic modifications in the cerebellar cortex of the adult rat persist for at least four weeks. J. Neurosci., 17(2), 717-721.

Klein, A., Wessolleck, J., Papazoglou, A., Metz, G.A. \& Nikkhah, G. (2009) Walking pattern analysis after unilateral 6-OHDA lesion and transplantation of foetaldopaminergic progenitor cells in rats. Behav. Brain. Res., 199(2), 317-325.

Laforce, R., Jr \& Doyon, J. (2002) Differential role for the striatum and cerebellum in response to novel movements using a motor learning paradigm. Neuropsychologia, 40(5), $512-517$.

Matsumura, M., Sadato, N., Kochiyama, T., Nakamura, S., Naito, E., Matsunami, K., Kawashima, R., Fukuda, H. \& Yonekura, Y. (2004) Role of the cerebellum in implicit motor skill learning: A PET study. Brain. Res. Bull., 63(6), 471-483. 
Metz, G.A., Antonow-Schlorke, I. \& Witte, O.W. (2005) Motor improvements after focal cortical ischemia in adult rats are mediated by compensatory mechanisms. Behav. Brain. Res., 162(1), 71-82.

Metz, G.A., Farr, T., Ballermann, M. \& Whishaw, I.Q. (2001) Chronic levodopa therapy does not improve skilled reach accuracy or reach range on a pasta matrix reaching task in 6-OHDA dopamine-depleted (hemi-Parkinson analogue) rats. Eur. J. Neurosci., 14(1), 27-37.

Metz, G.A. \& Whishaw, I.Q. (2002) Cortical and subcortical lesions impair skilled walking in the ladder rung walking test: A new task to evaluate fore- and hindlimb stepping, placing, and co-ordination. J. Neurosci. Methods, 115(2), 169-179.

Molinari, M. \& Petrosini, L. (1993) Hemicerebellectomy and motor behaviour in rats. III. Kinematics of recovered spontaneous locomotion after lesions at different developmental stages. Behav. Brain. Res., 54(1), 43-55.

Monfils, M.H., Plautz, E.J. \& Kleim, J.A. (2005) In search of the motor engram: Motor map plasticity as a mechanism for encoding motor experience. Neuroscientist, 11(5), 471-483.

Nixon, P.D. \& Passingham, R.E. (2001) Predicting sensory events. The role of the cerebellum in motor learning. Exp. Brain. Res., 138(2), 251-257.

Prchal, A., Albarracin, A.L. \& Decima, E.E. (2004) Blockage of vibrissae afferents: I. Motor effects. Arch. Ital. Biol., 142(1), 11-23.

Remple, M.S., Bruneau, R.M., VandenBerg, P.M., Goertzen, C. \& Kleim, J.A. (2001) Sensitivity of cortical movement representations to motor experience: Evidence that skill learning but not strength training induces cortical reorganization. Behav. Brain. Res., 123(2), 133-141.

Shin, J.C. \& Ivry, R.B. (2003) Spatial and temporal sequence learning in patients with Parkinson's disease or cerebellar lesions. J. Cogn Neurosci., 15(8), 1232-1243.

Smith, J.G. \& McDowall, J. (2006) The implicit sequence learning deficit in patients with Parkinson's disease: A matter of impaired sequence integration?. Neuropsychologia, 44(2), 275-288.

Stempowski, N.K., Carman, H.M. \& Fountain, S.B. (1999) Temporal phrasing and overshadowing in rat serial-pattern learning. Learn. Motiv., 30, 74-100.

Wallace, D.G. \& Fountain, S.B. (2002) What is learned in sequential learning? An associative model of reward magnitude serial-pattern learning. J. Exp. Psychol: Anim. Behav. Process., 28(1), 43-63.

Wallace, D.G., Rowan, J.D. \& Fountain, S.B. (2008) Determinants of phrasing effects in rat serial pattern learning. Anim. Cogn., 11(2), 199-214.

Webb, A.A. \& Muir, G.D. (2003) Unilateral dorsal column and rubrospinal tract injuries affect overground locomotion in the unrestrained rat. Eur. J. Neurosci., 18(2), 412-422.

Whishaw, I.Q. (2000) Loss of the innate cortical engram for action patterns used in skilled reaching and the development of behavioral compensation following motor cortex lesions in the rat. Neuropharmacology, 39(5), 788-805.

Whishaw, I.Q. (2005) Prehension. In: I.Q. Whishaw and B. Kolb, ed. The Behavior of the Laboratory Rat: A Handbook with Tests. New York: Oxford University Press, pp. $162-170$.

Whishaw, I.Q. \& Tomie, J.A. (1988) Food wrenching and dodging: A neuroethological test of cortical and dopaminergic contributions to sensorimotor behavior in the rat. Behav. Neurosci., 102(1), 110-123. 
Whishaw, I.Q. \& Tomie, J.A. (1989) Olfaction directs skilled forelimb reaching in the rat. Behav. Brain. Res., 32(1), 11-21.

Whishaw, I.Q., O'Connor, W.T. \& Dunnett, S.B. (1986) The contributions of motor cortex, nigrostriatal dopamine and caudate-putamen to skilled forelimb use in the rat. Brain, 109(Pt 5), 805-43.

Whishaw, I.Q., Pellis, S.M., Gorny, B.P. \& Pellis, V.C. (1991) The impairments in reaching and the movements of compensation in rats with motor cortex lesions: An endpoint, videorecording, and movement notation analysis. Behav. Brain Res., 42(1), 77-91.

Whishaw, I.Q., Li, K., Whishaw, P.A., Gorny, B. \& Metz, G.A. (2003) Distinct forelimb and hind limb stepping impairments in unilateral dopamine-depleted rats: Use of the rotorod as a method for the qualitative analysis of skilled walking. J. Neurosci. Methods, $\mathbf{1 2 6}(1), 13-23$.

Wolpaw, J.R. (1997) The complex structure of a simple memory. Trends Neurosci., 20(12), 588-594.

Wolpaw, J.R. \& Chen, X.Y. (2006) The cerebellum in maintenance of a motor skill: A hierarchy of brain and spinal cord plasticity underlies H-reflex conditioning. Learn. Mem., 13(2), 208-215. 